\title{
Face Recognition for Attendance Management
}

\author{
M Jaya Bhaskar ${ }^{1}$, Y Venkatesh ${ }^{2}$, R Sai Bhaskar Pranai ${ }^{3}$, M Rohith ${ }^{4}$. \\ ${ }^{1}$ Koneru Lakshmaiah Educational Foundation, India, jayabhaskar@kluniversity.in. \\ ${ }^{2}$ Koneru Lakshmaiah Educational Foundation, India, venkateshyaganti006@gmail.com. \\ ${ }^{3}$ Koneru Lakshmaiah Educational Foundation, India, rayaprolusaibhaskarpranai@gmail.com. \\ ${ }^{4}$ Koneru Lakshmaiah Educational Foundation, India, rohith.mallampalli@gmail.com
}

\begin{abstract}
Uniqueness or distinction of an individual is his face. Right now, of an individual is utilized with the end goal of participation making consequently. Maintaining participation is very critical for student success tests at all learning institutes, Uniqueness or distinction of an individual is his face. Right-now of an individual is utilized with the end goal of participation making consequently attendances are manually taken using unit leaders provided appointment sheets as part of the regulations. Expect that the term for one subject is around an hour or 1 hour and to record participation takes 5 to 10 minutes. For each guide this is utilization of time. This method is recursive, extremely particular but mistaken by certain understudies sign up regularly with lost mates. The strategy in fact, it allows it impossible to track participant involvement understudies just one enormous homeroom condition. To avoid these misfortunes, a programmed procedure is utilized right now depends on picture preparing. Right now, location and face acknowledgment is utilized. Right now, propose the structure and utilization of a face identification and acknowledgment framework to naturally identify understudies going to a talk in a homeroom and imprint their participation by perceiving their appearances.
\end{abstract}

Key words: Affirmation, Classifier, Enlishment, Face Recognition, Haar-Cascade.

\section{INTRODUCTION}

Keeping up participation is significant in complete known establishments to test the presentation on understudies. I participant attendance figures are collected remotely in most learning institutions using absence cards provided by the heads of division as a significant element of policy. In such sheets the understudies register and are then filled in or signed manually onto a Computer for potential examination. This system studios, some students register for their missing colleagues sometimes time consuming and unreliable. The technique likewise shapes it hard for following the participation like singles understudies where the huge study hall condition [1]-[5]. Throughout this research, will suggest the creation for the use of a facial identification and recognition device to instantly identify children attending a class lecture by identifying their faces and labeling their attendance. Although other authentication approaches (such as iris scans or fingerprints) might be more reliable, at the time they reach the classroom students typically have to queue for long. This (facial) biometric system consists of an enrollment process in which the specific features of a person's face are registered in a database, and then the recognition and authentication procedures. Face detection is preferred because of its non-intrusive existence and popularity, because people remember other individuals mainly based on their facial characteristics. In such, the face detected in a picture (obtained from the camera) is equated to the faces earlier stored captured at the period of enrollment. When unique bio metric character systems (including iris sweeps or fingerprints) may be additional accurate, at the time they enter the school room, assistants should regularly line for a long time. Look identification is selected inferably by its non-meddling existence and acknowledgement as individuals mainly capture specific persons who are essentially based on their facial highlights. Such biometric (facial) technology would introduce an enlistment system in which the outstanding highlights of a people's face will be inserted into a database into which the techniques for identifiable evidence and screening will be placed. In these, the recognized face in a picture (got from the camera) will be in examination with the once in the past put away faces caught at the hour of enlistment.

\section{LITERATURE SURVEY}

A face Detector needs to say only a picture by discretionary $h$ height includes a friendly face because whether that's all it is. Object detection may be done based on some indications: body coloring (of face in coloring photos and videos, movement (of heads in videos), face / face type, mannerism or a combination of those criteria [2]. Many estimates to facial exploration were focused in presence, even considering specific indications. Each section pane tests the image of the details at certain possible locations or sizes. Head position works when sorting a figure as a facial or just a semi-face from the component pane. That head / semi-face clustering algorithm is learned through head or head planning frameworks that utilize observable memorizing 
facts. Much of today's equations rely along the exploration framework of both the Viola Jones entity, that relies on Haar Cascades [3]-[4]. Hair-like main points of info were rectangular instances. The course is a Haar-like highlights advancement which is formed to structure an algorithm. A hair Fourier transform is a science ability which generates production of a cubic wave.

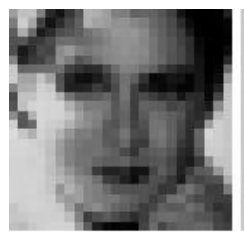

(a)

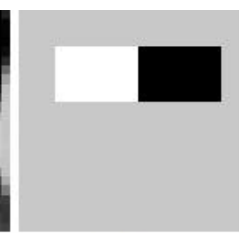

(b)

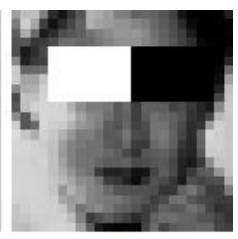

(c)
Figure 1: Hair alike Characteristics

Figure 1. Hair like Characteristics highlights, the foundation of a layout like (b) is colored dark to feature the example's help [4]. Just those pixels set apart in dark or white are utilized when the comparing highlight is determined.

\section{METHODOLOGY}

Right now, related segments regarding usefulness have been assembled to frame sub-frameworks which at that point consolidate to make up the entire framework. Separating the framework to segments and sub-frameworks illuminates the consistent plan regarding the class participation framework.

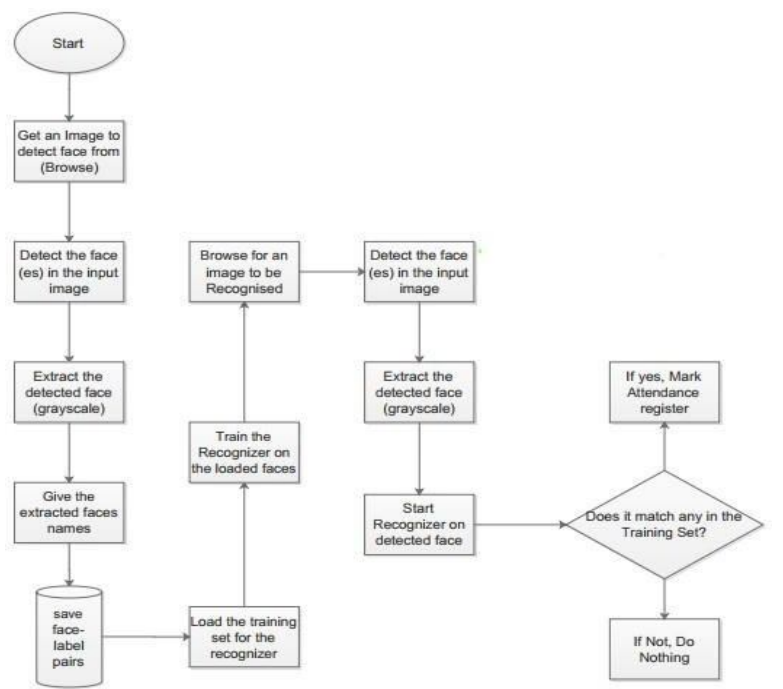

Figure 2: Process Order in classroom attendance system.

From Figure 2, It's very simple to see how a large portion of the sections used are comparable (the Image Securing section for the perusing of Source photos, Identity Identification and Identity Database to delete Feature Naming Ranges being during the stages of the facial detection cycle. The legitimate plan of the preparation set administration sub-framework is going to comprise of a picture securing part, a face discovery segment and a preparation set administration segment. Together, these parts cooperate database faces to handle preparation set. These will be executed in the context of a windows program. The Face Lexer consists of the image contracting part, the face lexer as well as the face classification segment, all of which work with the Face repository. Right now, the segment of acquiring and face detection is comparable to others in the post-framework of the Training Set Director as the utility is the closest approximation. The principal difference is the role of the face lexer and its regulation of the UI. It will load the set training again with the aim of prepari ng the lexer on the included faces and presenting the deci ded faces and usual face. For example, the face regexp system may consist of two important portions, the preparedness system supervisor as well as the eye regexp. Such dual sections will connect their looks collection, image secure, and face locator modules since they are normal in their utility. Furthermore, we must split the architecture into two modules and will have valid proposals for its nuts and bolts. The following mechanisms can be used to incorporate the standardized system.

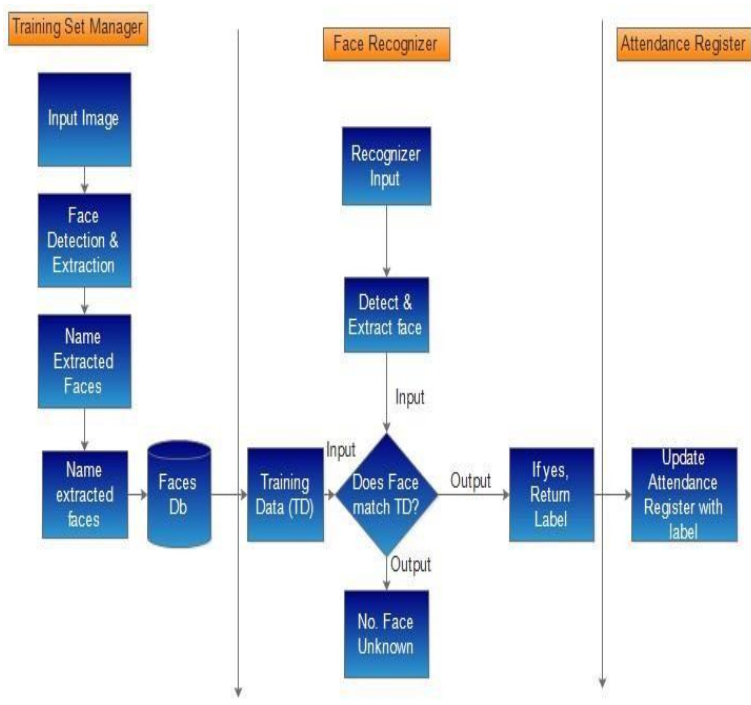

Figure 3: The logical view of the Desktop Module System

We were classified into two definitions: phones and tablets and computer devices [7]. The portable instruments are the parts that will assist with Handheld Module execution. Such a unit was accountable for picture acquisition of the understudies by study hall condition but also subsequently putting them away by the work area module for further preparation. That work area tools are sections; equipment but programming to be used to develop the work area module in real terms. Additionally, the work area node interfaces with category participation registry that executes the board framework as just a repository.

\section{EXPERIMENTAL INVESTIGATION}

This mission helps to robotize the omnipresent boring and time-squandering, traditional methods for stamping understudy involvement in study halls. The use of 
programmed participation by face discovery and recognition would improve the appropriateness of participation observation and of the board. This technique could also be used in evaluation corridors to monitor pantomime incidents, as the system will have the option of finding the shams that were not caught in the course of the registration procedures. Facial detection uses spread vastly where zones like suspect identifiable facts, safety systems, picture and video handling. This Program can also be discovered requirements in fully approved control agencies. We are creating to prepare a two-block system. The key element (facial indicator) is indeed a flexible component that is essentially a webcam program it catches the understudy heads, later secures on record using head regex calculations and facial take-out methods for desktop view. Its corresponding element is now a work area program that faces identification of the captured images (names) in the document, denotes the understudies record as well as saves the findings for future analysis in a database afterwards.

\section{IMPLEMENTATION}

A general framework of facial recognition fuses different methods: Facial recognition Highlight retrieval and Face recognition [7]. Eye discovery and recognition fuses different demonstrable elements when each element reflects an addition to following. Depends on the default framework and each component could function independently. Head distinguishing proof is indeed a Computer development which relies on figurines for studying can allocate faces for cutting edge images. Discovery of the face collects images film progressions for data it explores facial areas inside these photos. It is achieved via distinguishing facial from non-facial organi zation regions. Head details retrieval identifies major features inside a known face (eyebrows, jaw, nose, and facial sanctuaries). An unmistakable central problem to facial confirmation was the enormous differentiations here between comparable person's face pics as they stand out of different individuals. Currently, it is important to select a suitable face request program that can provide an average discrete limit between individuals. Facial lexer had broad range of usages. Because it provides an un-intrusive path of unmistakable confirmation from humans, a head was being used in safety systems as a major authentication. Gary Bradsky launched the OCV-Python at Intel in the year 1999 [9]. Earlier in the year 2000 the main action came to certain stage. OCV refers basically to open source computer vision library. It has interfaces similar to $\mathrm{C}++$ for Python and Java irrespective of how it is written in modified C / C++ [10]. OCV gloats about a working clientele wherever it rises bit by bit throughout the country due to the explosion of Computer perception technologies. OCV-Python is the OCV python application program interface. One may think of it as a python framework over the use of OCV on C++. It's not just quick to OCV-Python, but then again, it's anything but hard to code and move on [10]. This opts for a remarkable alternative in carrying out algorithmically elevated tasks. The basic explanation of the matter of face lexer in Computer perception could be organized as searches for pose either clip images from a plot line, perceiving nor testing by any rate single person at plotline using the set aside identity register. All things considered include two levels of facial recognition: facial identification in which a object is searched for in order to find a head, by then the image is set up to capture and focus the face of the person for easier identification. Face lexer that perceived and cared for face appears differently in relation to a recognized faces collection, to select whoever the person is. From 2002, face recognition could be done sensitively effectively what's more, reliably with the open source system of Intel named OCV. Face Detector which functions between 90-95 percent away from a camera-looking person.

\section{DETECTION CLASSIFIERS}

A Computer program that picks whether an image either a good (head image) or bad (non-head image) picture either classified by an algorithm. Computer program that determines whether an image is a good image (head image) or a bad image (non-head image) is known as a classifier [8]. In order to make sense of how to organize another image effectively, an algorithm was set up large count facial and semi-face images [6]. OCV equips us with two circa arranged and fit for facially recognizable evidence classificators

- Haar Classifier

- Cascaded Classifier

\section{a. Haar Classifier}

In specifics, Haar like highlights were cuboidal instances. structure the algorithm. The Haar perceptron was the researcher specific power which generates result of squared wave format. This is a non-interfering course for mankind unmistakable confirmation; the facial data is used as critical biometric applications in protection. OCV-Python is available in Intel by end of $20^{\text {th }}$ century by Gary Bradsky. A chief release worked to some degree by early 2000's. OCV essentially speaks to OCV. Open CV gloats about a working customer base wherever it goes all over planet, and this's use extending bit-bit Owing to Computer visual systems flooding Python or Java close by $\mathrm{C}++$, no matter how it's written in revamped $\mathrm{C} / \mathrm{C}++$. You may think of it as a py module in the use with OCV in $\mathrm{C}++$. Simply snappy yet then again is anything besides hard to code. One Haar include is anyway insufficient as there are a few highlights those coordinate compressed memory black and white zones at the foundation of the picture of figure 2.4 it is known as a feeble classifier. Haar falls, the premise of Viola Jones location system along these lines comprise of a progression of frail classifiers whose exactness is at any rate half right. If a territory goes through a full algorithm, this passes for later powerless algorithm

\section{b. Cascaded Classifier:}

Include classifier accomplishes $100 \%$ facial location rate and having half bogus supportive growth. A 5-include distribution accomplishes $100 \%$ discovery the chage and double the $20 \%$ bogus good change (2X10\% combined). A 10+10 -component algorithm 
accomplishes cent percent recognition change of double the $5 \%$ bogus supportive growth (double the one percent cumulative). Adding a few feeble distributions develops exactness of location.

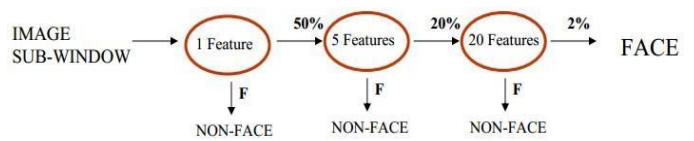

Figure 4: Several classifiers for face recognition enhancement

After the last course had been developed, the prerequisite is set of an approach for rapidly process Haar highlights, let instance process out distinctions at separate regions. Basic picture is materialistic right now, picture. Number of initial picture values can be determined inside the box [4] If D, C, B, A are estimates for basic picture that edges in a square shape which is shown below.

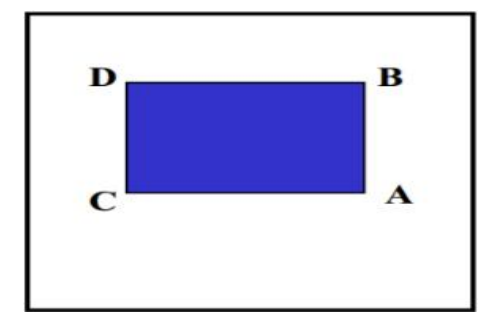

Figure 5: scales for integrated picture on square.

$$
\text { Sum }=\mathrm{A}-\mathrm{B}-\mathrm{C}+\mathrm{D}
$$

The square shape requires just three increases for any size. This approach to face discovery limits calculation time while achieving high exactness of the location. Currently it is used in many PC vision zones.

\section{RESULT AND DISCUSSION}

The last preliminary type was facial-detection structure that relies upon OCV. Results by work are shown below with using interactive module, in which a head picture is identified.

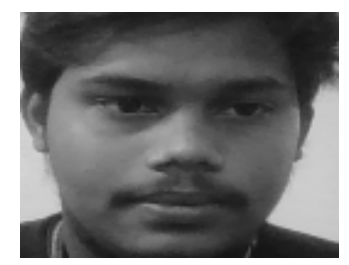

Figure 6: Example of Face Detection.

Considering the examination of the submitted OCV, it was established that there are no single techniques and headways to create a scattered information structure of affirmation which would join all periods of system advancement. There are a huge number of strategies to look for, arrange and sift through the technique of affirmation. By choosing advances, you should be amazingly careful considering how you should use certain methodologies depending on your needs. Operating along these lines is currently important with a quick and dirty representation of affirmation progressions, likewise, as developing new advances and a way to deal with this pressing issue. As mentioned above, PC vision and face-recognition gadgets are successfully developed by and through. Overall development pioneers put a lot of money into imaginative work as this issue has open entrances for usage all the way through. The course to boost face affirmation is largely through the expanded validation schemes.

\section{CONCLUSION}

This may very well be concluded that the executive system has been developed to supplant a manual and problematic structure with a strong, stable, fast and efficient class involvement [1]. A face-to-face research and acknowledgement process would save resources, the the organization's job assessment and supplant the paperwork content now being utilized for successful electronics equipment. There's no prerequisite for the implementation of the system for different hardware because it only requires a Computer and lens. Subsequently, the lens takes a considerable task in a function of the system as well as the image quality and operation of the lens circumstance should be increasingly attempted especially unless the shot is operated from a live webcam stream. The framework can likewise be utilized in consent-based frameworks and secure access verification (limited offices) for get to the board, home video reconnaissance frameworks for individual security or law authorization. The significant danger to the framework is Spoofing. A system may be used to get to the table of approval-based systems as well as safe entry identification (restricted agencies), digital video identification mechanisms for citizen compliance, or law authorization. Phishing is the big danger to the system. For example, 83.1 per cent of human canonization can be termed at from the general usefulness of the system to render the construct idiot proof. Subsequently, a module could be implemented which documents all unspecified eyes gleamed, and the instructor may answer them psychologically. Future work Might also provide a few tightly structured involvements lists of each classroom but also the opportunity to produce pay period-to-month engagement updates and then send them to suitable personnel audit.

\section{REFERENCES}

1. V. Shehu and A. Dika, Using Real Time Computer Algorithms in Automatic Attendance Management Systems. IEEE, pp. 397 - 402, Jun. 2010.

2. Prof. P.K Biswas, Digital Image Processing.

3. S. Z. Li and A. K. Jain, Eds., Handbook of face recognition. New York:Springer, 2005.

4. N. Mahvish, Face Detection and Recognition, Few Tutorials, 2014. 
5. Anil K Jain, Lin Hong, Sharath Pankanti, and Ruud Bolle, Biometric Identification. IEEE, 2004.

6. N. Tom, Face Detection, Near Infinity - Podcasts, 2007.

7. T. Kanade, Computer recognition of human faces. Basel [etc.]: Birkhäuser, 1977. https://doi.org/10.1007/978-3-0348-5737-6

8. Tun Tun Oo, Aaron Don M.Africa, Emotion Basic action using Face Recognition and Detection ANN, IJETER,NOV 2019.

9. Nishad Nawaz, Artificial Intelligence Face Recognition for Applicant Tracking System, IJETER, DEC 2019. https://doi.org/10.2139/ssrn.3521931

10. J.D. Pujari, Dashrarth.K.Bhadangkar, Rjesh. Yakkundimath, Identification and Recognition of Facial Expressions Using Image Processing Techniques: A Survey, IJETER, MAY 2019. 